\title{
ON MATROIDS ON EDGE SETS OF GRAPHS WITH CONNECTED SUBGRAPHS AS CIRCUITS
}

\author{
J. M. S. SIMÕES-PEREIRA ${ }^{1}$
}

ABstract. It is proved that if $\mathscr{F}$ is a finite family of connected, finite graphs, then a graph $G$ exists such that the subgraphs of $G$ isomorphic to a member of the family cannot be regarded as the circuits of a matroid on the edge set of $G$.

1. In a recent paper [1] we have proved that there are only two matroids on the edge set of any graph $G$ (let us call them edge set matroids), whose circuits are connected subgraphs which form homeomorphic equivalent classes. These matroids are the polygon-matroid, whose circuits are the cycles, and the matroid of bi-circular subgraphs, where a bi-circular graph is a graph formed by two cycles which either have a path in common, or a vertex in common, or are disjoint but linked by a path; these graphs are homeomorphic to those pictured in Figure 1.
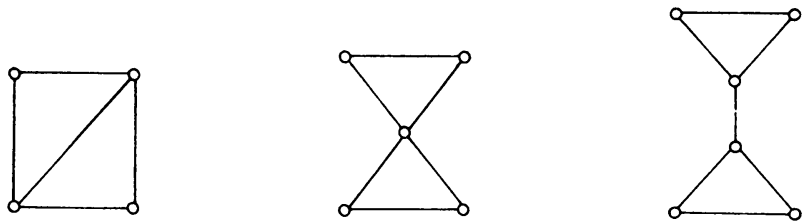

FIGURE 1

The hypothesis concerning homeomorphism is essential to the arguments in [1]. If we drop this hypothesis, the problem of finding all edge set matroids seems to be a very difficult one. As an unknown referee pointed out to me, a matroid of this kind is the matroid whose circuits are: (i) all cycles of even length; (ii) all graphs consisting of two cycles

\footnotetext{
Received by the editors July 21, 1972.

AMS (MOS) subject classifications (1970). Primary 05B35; Secondary 05 C 99.

Key words and phrases. Matroid, graph, matroid circuit, subgraph, subgraphs as matroid circuits.

${ }^{1}$ Supported by a scholarship from the Deutscher Akademischer Austauschdienst and by a grant from Instituto de Arta Cultura, Lisboa, Portugal. Research done at the Mathematisches Institut der Technischen Universität München. Thanks are due to the referee for comments on a previous version of this paper.
}

c) American Mathematical Society 1973 
of odd length, having only one vertex in common; (iii) all graphs consisting of two cycles of odd length, joined by a path. In any graph $G$, the subgraphs of these kinds are the circuits of a matroid on the edge set of $G$ but a cycle of odd length, although homeomorphic to a cycle of even length, is not a circuit of the matroid.

In this note we prove a theorem concerning edge set matroids. Our terminology is now slightly different from that used in [1]: we reserve the word "circuit" for the matroid-circuits and use "cycle" for simple closed paths in a graph. Moreover a matroid is defined as follows (see Whitney [2]):

Let $E$ be a set of elements and $\mathscr{K}$ a family of subsets of $E$ (circuits). $\mathscr{K}$ defines a matroid on $E$ if and only if the following axioms hold:

Ахгом 1. No circuit is properly contained in another circuit.

Aхıом 2. If $K$ and $K^{\prime}$ are distinct circuits, $a \in K \cap K^{\prime}$ and $b \in K^{\prime}-K$, then a circuit $K^{\prime \prime}$ exists such that $b \in K^{\prime \prime} \subset K \cup K^{\prime}-\{a\}$.

2. Let $\mathscr{F}$ be a family of abstract connected graphs such that in any graph $G$ the subgraphs isomorphic to members of $\mathscr{F}$ are the circuits of a matroid. Call the members of $\mathscr{F}$ circuits. Then

Lemma 1. No circuit has a pendant edge.

Proof. Let $K$ be a circuit with a pendant edge, say $x$. Take another circuit $K^{\prime}$, equal to $K$, and let $K \cup K^{\prime}$ be such that $x$ is the only edge common to $K$ and $K^{\prime}$ and the pendant vertex of $x$ in each circuit coincides with the vertex of higher degree in the other circuit. Clearly, $x$ is a bridge in $K \cup K^{\prime}$. By Axiom 2, $K \cup K^{\prime}-\{x\}$ contains a circuit. But since all circuits must be connected, the existence of such a circuit contradicts Axiom 1.

Thus the lemma is proved.

THEOREM 1. Let $\mathscr{F}$ be a finite family of connected, finite graphs. Then a graph $G$ exists such that the subgraphs of $G$ isomorphic to a member of $\mathscr{F}$ (or, for brevity's, sake belonging to $\mathscr{F}$ ) cannot be regarded as the circuits of a matroid on the edge set of $G$.

Proof. Let $\mathscr{F}$ be a finite family of finite, connected graphs. The members of this family may eventually be regarded as the circuits of a matroid on the edge set of some graphs. However a graph $G$ always exists with a subgraph which, according to the definition of a matroid, must also be a circuit but which does not belong to the family. This is a consequence from the fact that, for the members of a family $\mathscr{F}$ of connected, finite graphs to be circuits of an edge-set matroid defined on any graph $G$, there must always exist a member of $\mathscr{F}$ with a pair of edges of minimal distance arbitrarily large. 
To prove it let $K$ be a circuit, $\alpha=\left(a_{1}, a_{2}\right), \beta=\left(b_{1}, b_{2}\right)$ two edges of $K$. Consider the four distances $d\left(a_{i}, b_{j}\right)$ for $i, j=1,2$. Let $r$ be the minimal distance between $\alpha$ and $\beta$, and suppose we choose a pair $\alpha, \beta$ in $K$ for which this distance is maximal among all edge pairs. Moreover, without loss of generality, we may suppose $d\left(a_{1}, b_{1}\right)=r$. There are 6 distinct cases which are summarized in Table I (columns 1 to 5).

$\begin{array}{rcccccccc}\text { Cases } & d\left(a_{1}, b_{1}\right) & d\left(a_{1}, b_{2}\right) & d\left(a_{2}, b_{1}\right) & d\left(a_{2}, b_{2}\right) & d\left(a_{1}, b_{1}\right) & d\left(a_{1}, b_{2}\right) & d\left(a_{2}, b_{1}\right) & d\left(a_{2}, b_{2}\right) \\ \text { I } & r(>0) & r & r & r & r & r+1 & r & r+1 \\ \text { II } & r(>0) & r+1 & r & r & r & r+1 & r & r+1 \\ \text { III } & r(>0) & r+1 & r & r+1 & r+1 & r+1 & r+1 & r+1 \\ \text { IV } & r(>0) & r+1 & r+1 & r & r & r+1 & r+1 & r+1 \\ \text { V } & r(\geqq 0) & r+1 & r+1 & r+1 & r & r+1 & r+1 & r+2 \\ \text { VI } & r(\geqq 0) & r+1 & r+1 & r+2 & r+1 & r+1 & r+2 & r+2\end{array}$

TABLE I

Take another circuit $K^{\prime}$ and let $K \cup K^{\prime}$ be such that $\beta$ is the only edge common to $K$ and $K^{\prime}$. For simplicity let us say the edges of $K$ are black and those of $K^{\prime}$ are blue. Now, by Axiom 2, a circuit $K^{\prime \prime}$ exists such that $\alpha \in K^{\prime \prime} \subset K \cup K^{\prime}-\{\beta\}$. By Axiom 1, $K^{\prime \prime}$ contains both black and blue edges. Since $K^{\prime \prime}$ must be connected and as a consequence of Lemma 1, either $K^{\prime \prime}$ contains at least one blue path $P\left(b_{1}, b_{2}\right)$ with length $s \geqq 2$, or at least one of the vertices $b_{1}$ and $b_{2}$ is a cut-point of $K^{\prime \prime}$ and there exists at least one cycle in the blue block of $K^{\prime \prime}$ relative to this cut-point.

If blue paths exist, then take one with minimum length $s \geqq 2$. We distinguish two possibilities:

(a) $s \geqq 3$. Let $\beta^{\prime}$ be an edge of $P\left(b_{1}, b_{2}\right)$ incident to neither $b_{1}$ nor $b_{2}$. The minimal distance between $\alpha$ and $\beta^{\prime}$, which both belong to $K^{\prime \prime}$, is $\geqq r+1$, that is to say, we obtain a new circuit $K^{\prime \prime}$ from a given circuit $K$ with a pair of edges $\alpha$ and $\beta^{\prime}$ whose minimal distance is greater than the minimal distance between the edges $\alpha$ and $\beta$ of $K$.

(b) $s=2$. Let $\left(b_{1}, x\right),\left(x, b_{2}\right)$ be the edges in $P\left(b_{1}, b_{2}\right)$. We have to examine the 6 cases of Table I. In cases I, II, IV and V, we set $\beta^{\prime}=\left(b_{1}, x\right)$ and $x$ plays now the role of $b_{2}$. In cases III and VI, we set $\beta^{\prime}=\left(x, b_{2}\right)$ and $x$ plays the role of $b_{1}$. The new distances between the endpoints of $\alpha$ and $\beta^{\prime}$ are given in the columns 6 to 9 of Table I. With this operation we obtain, in cases III and VI, a pair of edges in $K^{\prime \prime}$, namely $\alpha$ and $\beta^{\prime}$, whose minimal distance is larger than the distance between $\alpha$ and $\beta$. In the remaining cases, to obtain a circuit with a pair of edges satisfying this condition, one or two iterations of this operation may be required, each time with $K^{\prime \prime}$ and $\beta^{\prime}$ in the roles of $K$ and $\beta$, respectively. In fact, cases I and II yield case III, case V yields case VI and case IV yields in a first iteration case V 
which in turn yields case VI. Now from cases III and VI, a new iteration allows us to achieve our aim.

If no blue path exists, then take the above mentioned blue cycle. Suppose the cycle belongs to the blue block of $b_{1}$. (The same argument holds a fortiori with $b_{2}$ instead of $b_{1}$.) Let $\beta^{\prime}$ be an edge of the cycle nonincident to $b_{1}$. Obviously, the minimal distance between $\alpha$ and $\beta^{\prime}$ is $\geqq r+1$.

Hence it is always possible to obtain from a pair of edges $\alpha, \beta$ in a circuit $K$, whose distance is $r$, a new pair $\alpha, \beta^{\prime}$ in a circuit $K^{\prime \prime}$, whose distance is $\geqq r+1$. By repeating the argument, the theorem is proved.

Theorem 1 may also be stated more briefly as follows.

THEOREM 1'. No edge-set matroid (on an arbitrary graph) may exist with a finite number of connected, finite graphs as circuits.

\section{REFERENCES}

1. J. M. S. Simões-Pereira, Subgraphs as matroid cells, Math. Z. 127 (1972), 315-322.

2. H. Whitney, On the abstract properties of linear dependence, Amer. J. Math. 57 (1935), 501-533.

Secção de Matemática, Universidade de Coimbra, Coimbra, Portugal 